or polymerase chain reaction, is effectively a way of photocopying DNA and has become an essential tool for geneticists.

The work by Clevers helped to make the case that CRISPR-Cas9 is not just a tool of basic science, but a source of medical breakthroughs to come. The CRISPR craze is now in full swing, and the platform's ability to treat a range of diseases - from severe combined immunodeficiency (SCID) to muscular dystrophy - is being put to the test. Many of the scientists involved predict that its medical applications will rapidly outstrip those of the other main genome-editing tools, such as transcription activator-like effector nucleases (TALENs) and zinc-finger nucleases (ZFNs), because CRISPR-Cas9 is more efficient and easier to use.

The consensus is that monogenic diseases those involving only one gene - are the lowhanging fruit of the field. But even the most ardent genome-editing enthusiasts say that this term is misleading. "The fruit is still pretty far up the tree," says Chad Cowan, a stem-cell biologist at Harvard University and co-founder of CRISPR Therapeutics, a biotech company based in Cambridge, Massachusetts, set up to use CRISPR-Cas9 to cure diseases.

There are many factors that determine whether genome editing is a viable approach for a particular disease. The main difficulty - the one that dictates which diseases are plausible targets for therapeutics - is delivering the therapy, and this strongly depends on the ability to access the cells or organs that need correction. But many characteristics guide researchers in prioritizing their efforts. The percentage of cells whose genomes must be edited to achieve a medical benefit is one important factor, as is whether treating the affliction requires deleting, introducing or correcting genes.

\title{
REMOVING THE PROBLEM
}

The delivery hurdle is so substantial that researchers are trying to work around it, rather than overcome it. One strategy is to extract cells, edit their genomes, check that there are no unintentional genetic changes, known as 'off-target effects', and then reintroduce them to the body so that they can operate as healthy cells. This approach is particularly promising for problems of blood and bone marrow, including HIV (see page S8) and sickle-cell disease.

Efforts are already underway to develop CRISPR-Cas9 treatments to tackle sicklecell disease. One of the painful symptoms of the disease is caused by misshapen blood cells clogging the blood vessels, and researchers hope that gene editing could offer a treatment, if not a cure. The target is a gene called BCL11A, which causes red blood

$\rightarrow$ NATURE.COM To read more on monogenic disorders visit:

go.nature.com/iadugc the gene that is disrupted in people with cystic fibrosis. This was one of the first attempts to remarkable how well CRISPR works," he says. "I've never seen anything - apart from PCR - that was so simple and so powerful." PCR, 
$\vec{a}$ cells to produce adult, rather than fetal, haemoglobin. Fetal haemoglobin does not form long chains, so tricking cells into producing it could result in less clogging by red blood cells. A team of researchers including Feng Zhang of the Broad Institute in Cambridge, Massachusetts, recently showed that using CRISPR-Cas9 to make cuts in the genomic region that controls the expression of $B C L 11 A$ increases the production of fetal haemoglobin ${ }^{1}$.

Sickle-cell disease is a straightforward target, even among monogenic diseases without problematic delivery, and viral diseases of the blood can be tackled in a similar way. Cowan's group has successfully used CRISPR-Cas9 to disable the CCR5 gene in half of the blood stem cells treated ${ }^{2}$. This is important because HIV uses the CCR5 receptor to enter cells (see page S8). Applying this approach to bone-marrow cells could effectively immunize people against the virus, he says.

"Inactivating a disease-causing gene is a whole lot easier than correcting a gene," says Erik Sontheimer, a biologist at the University of Massachusetts Medical School in Worcester and co-founder of Intellia Therapeutics, based in Cambridge, Massachusetts, which also develops CRISPR-Cas9-based treatments. However, there are not many heritable diseases that can be fixed by simply knocking a gene out, adds Bryan Cullen, a molecular geneticist at Duke University in Durham, North Carolina.

That said, diseases with a strong genetic component in which there is one healthy and one mutant gene variant are good candidates for this kind

of approach. In such cases, switching off the mutant variant allows the healthy copy of the gene to function properly, says David Segal, a genome researcher at

"Inactivating a disease-causing gene is a whole lot easier than correcting $a$ gene."

the University of California, Davis. Segal thinks that Huntington's disease, a neurodegenerative disorder caused by a single mutation, is a prime example of a disease that could benefit from this approach. The only problem - and it is a significant one - is that the cells in need of correction are not readily accessible because they are found in the brain.

The eye, however, presents a much easier target. Tara Moore, a molecular biologist at Ulster University in Belfast, UK, is using this strategy to treat Meesmann's epithelial corneal dystrophy, a heritable disease resulting in cysts on the cornea that can cause irritation and blurred vision. She has successfully used CRISPR-Cas9 to find and disable gene variants that cause the disease, leaving the healthy allele intact in cornea-generating stem cells ${ }^{3}$. She estimates that this approach

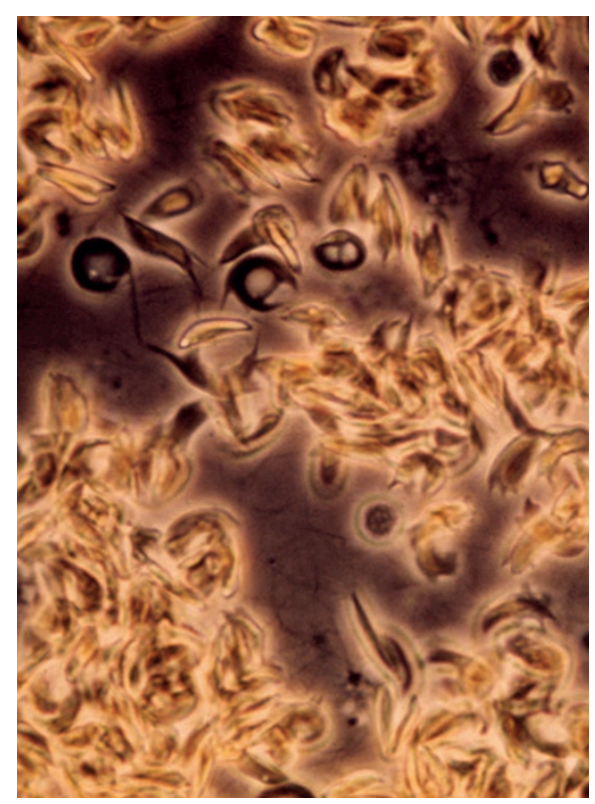

Blood cells are misshapen in sickle-cell disease.

could work in roughly one-third of the 76 mutations that are known to cause corneal disorders, of which Meesmann's is just one. These 76 mutations are spread among just four genes. "The eye is so accessible, and is such a small area to treat," she says. "And we're able to clearly monitor it to note improvement."

\section{INSERTING A SOLUTION}

An alternative to disrupting a gene is to introduce one, but that requires getting a DNA template to the site of genome editing (see page S2). This has been achieved for the liver, as a treatment for type I tyrosinaemia demonstrates. Those with this disease have a faulty gene called $F A H$ that reduces their ability to break down the amino acid tyrosine, resulting in liver damage. Last year, scientists at the Massachusetts Institute of Technology used CRISPR-Cas9 to insert a healthy version of the FAH gene into the liver cells of laboratory mice. The healthy gene was expressed in only 1 of every 250 liver cells, but this was enough to reduce liver damage 4 .

Perhaps not surprisingly, diseases that can be alleviated by editing only a small percentage of cells are among the first to be targeted. Like type I tyrosinaemia, SCID falls into this category, and it has a delivery advantage: cells with a healthy or corrected gene sequence proliferate once they are put back into the body. By comparison, most cancers probably require all of the relevant genes to be edited to stop the disease from rebounding. Other diseases in which correcting a small percentage of cells might make a big difference include glycogen storage disease and ornithine transcarbamylase deficiency, an inherited disorder that causes ammonia to accumulate in the blood. But researchers might be hesitant - ornithine transcarbamylase deficiency was at the centre of a gene-therapy trial in 1999 that was shut down after a patient died.

In comparison with CRISPR-Cas9, the older genome-editing tools, TALENs and ZFNs, can struggle with the task of correcting a gene, especially when it comes to achieving a normal level of gene expression from the edit. Sometimes overexpressing a gene can increase the risk of cancer. In this regard, says Cowan, "CRISPR-Cas has a defining and significant advantage over other gene-editing techniques."

But CRISPR-Cas9 is not always the optimal choice of the genome-editing tools available. When success comes down to size, for example, ZFNs often have a distinct advantage. Adenoassociated viral vectors, which are promising delivery systems, especially to the liver, can accommodate a ZFN as well as an engineered gene template ${ }^{5}$, but sometimes struggle to squeeze in the larger genome-editing tools, TALENs and CRISPR-Cas9. Moreover, CRISPR-Cas9 is known to have less inherent specificity than TALENs with their long DNA recognition domains - a particular concern for the editing of large and complex genomes - although researchers have the made great gains in reducing these 'off-target' effects.

Genome editors ultimately hope to tackle diseases of all levels of genetic complexity and affecting all parts of the body. But at the moment, even the therapies that are closest to approval are still a good way off. "Five years would be an aggressive timeline, but it could happen," says Cowan.

This does not stop researchers from dreaming. Segal, for example, suggests that the brain presents probably the most formidable challenge for delivering genome-editing therapies. But he is well aware that there are many singlegene neurological disorders: Angelman syndrome, Huntington's disease and Prader-Willi syndrome, to name just three.

In the short-term, the focus on diseases of the eye, blood and liver, which are the easiest organs to target with CRISPR-Cas9, will continue. Since his early success with cystic fibrosis, Clevers has started working on the liver, primarily because knowledge of how to transplant corrected stem cells - a key step in many putative genome-editing therapies - is more advanced for the liver than for the lung.

Cullen has the same instinct. "My bet is that the first successes of CRISPR-Cas9 treatments will involve diseases in the liver," he says. "The liver is where everything goes, whether you want it to or not."

Virginia Gewin is a freelance science writer based in Portland, Oregon.

1. Canver, M. C. et al. Nature http://dx.doi org/10.1038/nature15521 (2015)

2. Mandal, P. K. et al. Cell Stem Cell 15, 643-652 (2014).

3. Courtney, D. G. et al. Gene Therapy http://dx.doi. org/10.1038/gt.2015.82 (2015).

4. Yin, H. et al. Nature Biotechnol. 32, 551-553 (2014).

5. Gaj, T. et al. Trends Biotechnol. 31, 397-405 (2013). 